\title{
Causally Continuous Spacetimes
}

\author{
S. W. Hawking \\ Institute of Astronomy, University of Cambridge, Cambridge, UK. \\ R. K. Sachs* \\ DAMTP, University of Cambridge, and Departments of Mathematics and Physics, \\ University of California at Berkeley, Berkeley, USA
}

Received August 8, 1973

\begin{abstract}
Causally continuous general relativistic spacetimes are defined and analyzed. In a causally continuous spacetime, the past and future of a local observer behave continuously under small perturbations of the metric or small changes in his location. Causally simple spacetimes are causally continuous; causally continuous spacetimes are causally stable. Possible reasons for taking causal continuity as a basic postulate in macrophysics are briefly discussed.
\end{abstract}

\section{Introduction}

Throughout this paper we consider macrophysics and use a nonquantum, general relativistic, time-oriented spacetime to model physical spacetime. What global properties does spacetime have? There seem to be three main possibilities.

First, there might conceivably be causality violations, even at the macroscopic level. Some important models, such as certain Kerr metrics, do have self-intersecting causal curves $([3,9])$. But macroscopic causality violations would imply a drastic alteration of standard physics. As yet, we have neither empirical evidence nor compelling theoretical arguments that causality violations occur.

Now suppose macroscopic causality violations cannot occur. We should presumably use models which remain causal even if the spacetime metric is perturbed slightly, since quantum and other limitations mean our macroscopic models and measurements are imprecise. Formally, this means using stably causal spacetimes $([7,9])$. Stable causality is perhaps the most plausible global assumption to make.

Finally, it may be that we should work exclusively with stably causal spacetimes which obey further restrictions. For example, globally hyberbolic spacetimes have a global Cauchy surface and are stably causal; asymptotically simple and empty spacetimes have, in addition, a behavior "at infinity" similar to that of Minkowski space $([11,12,9])$. But observations, especially observations of the cosmological microwave background,

* Supported by grants from the NSF, SRC, and Guggenheim foundation. 
show that the universe as a whole is not asymptotically simple and empty $([8,9])$. Moreover, there are no convincing physical arguments for supposing a global Cauchy surface exists: even if one does exist we apparently have no way of knowing all the data it carries; in any case we seem to be continually and rather directly getting new information from the big bang. Many useful models are not globally hyperbolic ([9]).

In this paper we characterize and discuss a restriction we shall call causal continuity. Roughly, a causally continuous spacetime is stably causal, has no really big gaps (gaps of "dimension" more than 2), and is not too "concave" at infinity. For such a spacetime, the ideal point boundary of Geroch, Kronheimer, and Penrose [6] has a consistent causal structure ([2]) and a certain algebraic system (complete lattice) determined by the spacetime behaves continuously ([2]). Causal continuity is intermediate between stable causality and global hyperbolicity; it is a moderately plausible candidate for a fundamental restriction on macroscopic spacetime.

In Section 1 following we use a simple example to illustrate various "peculiar" features a spacetime can have. There may be observers who, intuitively speaking, can almost peek around infinity or a singularity but not quite. A small change in position or perturbation of the metric may have a drastic effect on pasts or futures. Judging how "late" an event is by the "size" of the region from which it can receive signals may lead to a discontinuous function.

In Section 2 we show, under appropriate restrictions, that a spacetime either has all these pecularities or none of then. We define a causally continuous spacetime essentially as one which has none of them. We prove that a causally simple spacetime ([9]) is causally continuous and that a causally continuous spacetime is causally stable. We list how causal continuity is related to various other global conditions that are sometimes used.

Though even our results are of mainly technical interest, we have tried to confine the most technical parts to the proofs and a footnote, which the reader may omit without esential loss of context.

\section{Preliminaries}

Throughout this paper, $M$ is a time-oriented spacetime whose metric $g$ has signature $(-,+,+,+)^{1}$.

\footnotetext{
1 We shall use the terminology and notation of Hawking and Ellis ([9]) and of Bishop and Goldberg ([1]). A spacetime is time-orientable iff there is an everywhere timelike vector field on it. A time-orientation can then be specified by designating one such vector field, say $X$, as future-directed. A $C^{1}$ curve $\phi: E \rightarrow M$ is then future-directed iff $g\left(\phi_{*} e, \phi_{*} e\right) \leqq 0$ and $g\left(\phi_{*} e, X \phi e\right)<0$ for all $e \in E$. Thus a future-directed $C^{1}$ curve is everywhere timelike or lightlike and its tangent vanishes nowhere. $M$ is causal iff each such curve is $1-1$.
} 


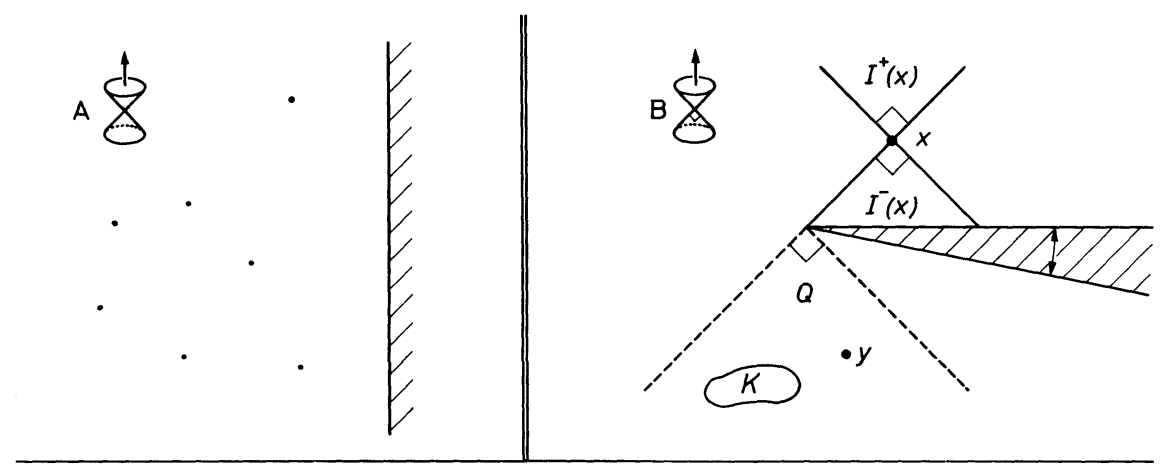

Fig. 1.2. The spacetime $A$ is reflecting; the spacetime $B$ is not

Let $S \cong M$ be a subset. The chronological past $I^{-}[S]$ of $S$ consists of those events which can signal to some observer in $S$ at a speed less than the speed of light. Formally, $I^{-}[S]=\{z \in M$ : there is a future-directed timelike curve from $z$ to some $s \in S\}$. Similarly, the causal past $J^{-}[S]$ of $S$ is defined by $J^{-}[S]=\{z \in M: z \in S$ or there is a future-directed curve from $z$ to some $s \in S\}$. If $S$ is open, its (chronological) common past $\downarrow S$ is the largest open set each event of which can signal to each observer in $S$ at a speed less than that of light. Formally, $\downarrow S=$ Interior $\{z \in M$ : for all $s \in S$ there is a future-directed timelike curve from $z$ to $s\}$. Unless $S$ is empty, $I^{-}[S] \supseteqq \downarrow S . I^{+}[S], J^{+}[S]$, and $\uparrow S$ are defined dually; dual results will often be taken for granted. If $z$ is an event, we abbreviate $I^{ \pm}[\{z\}]$ by $I^{ \pm}(z)$ and similarly for $J^{ \pm}$.

Proposition 1.1. For all $z, \downarrow I^{+}(z) \supseteqq I^{-}(z)$.

Proof. For all $z, I^{-}(z)$ is open and $I^{-}(z) \cong I^{-}(w)$ for all $w \in I^{+}(z)$ $([9,13])$. The proposition follows.

Call $M$ reflecting if the following condition holds: for all events $x$ and $y$ in $M, I^{+}(y) \supseteqq I^{+}(x)$ iff $I^{-}(x) \supseteqq I^{-}(y)$; if $M$ is causal this definition corresponds to the definition of past and future reflecting in Kronheimer and Penrose [10]. Fig. 1.2 shows one spacetime that is reflecting and one that is not. Each of the two spacetimes is conformal to a submanifold of 2-dimensional Minkowski space. The shaded regions represent closed subsets which have been amputated, say because the conformal factor, whose precise form is irrelevant here, blows up as one approaches the boundary. Similarly, the dots in Fig. 1.2.A represent missing points. More realistic models may have a similar behavior at singularities or at infinity. 
The spacetime 1.2. B is not reflecting since $I^{+}(y) \supseteqq I^{+}(x)$ but $I^{-}(x) \nsupseteq I^{-}(y)$. It has various related properties which might be regarded as unphysical. $y$ can send signals to events arbitrarily close to $x$, but $x$ cannot recieve any signals from within $Q$. It it occurs locally, such behavoir is pathological ([15]). In addition, $\downarrow I^{+}(x)$ contains the open set $Q$, so $\downarrow I^{+}(x)$ is much larger than $I^{-}(x)$. Moreover, a small change in location or small perturbation of the metric can result in a sudden increase of $I^{-}(x)$. And thus the "size" of $I^{-}(x)$ is much smaller than the size of the chronological past of some neighboring events. All these phenomena are interrelated. In the rest of this section we give some definitions and propositions useful in analyzing each phenomenon when one has a general time-oriented spacetime $M$. The next section discusses the interrelations in detail.

Proposition 1.3. The following conditions are equivalent.

A) $M$ is reflecting;

B) for all events $v$ and $w$ in $M, v \in$ Closure $\left[J^{+}(w)\right]$ iff $w \in$ Closure $\left[J^{-}(v)\right]$;

C) for all events $z$ in $M, \downarrow I^{+}(z)=I^{-}(z)$ and $\uparrow I^{-}(z)=I^{+}(z)$.

Proof. In any case Closure $\left[J^{ \pm}(z)\right]=$ Closure $\left[I^{ \pm}(z)\right]$ $=\left\{w \in M: I^{ \pm}(z) \supseteqq I^{ \pm}(w)\right\}([9,13])$. Thus condition A and condition B are equivalent. Now suppose $w \in \downarrow I^{+}(z)$; then $I^{-}(w) \subseteq I^{-}(v)$ for all $v \in I^{+}(z)$. If $M$ is reflecting, $I^{+}(w) \supseteqq I^{+}(v)$ for all such $v, v \in$ Closure $\left[I^{+}(w)\right]$ for all such $v$, thus $z \in$ Closure $\left[I^{+}(w)\right]$, and finally we Closure $\left[I^{-}(z)\right]$. Thus $\downarrow I^{+}(z) \cong$ Closure $\left[I^{-}(z)\right]$ if $M$ is reflecting. Now in any case Interior $\left\{\right.$ Closure $\left.\left[I^{-}(z)\right]\right\}=I^{-}(z)([9,13])$. This result, Proposition 1.1, and the dual argument show that condition $\mathrm{A}$ implies condition C. For the converse, suppose $I^{+}(w) \supseteqq I^{+}(v)$. Then $\downarrow I^{+}(v) \supseteqq \downarrow I^{+}(w)$ by the way in which common pasts were defined. If condition C holds, $I^{-}(v) \supseteqq I^{-}(w)$. Dually, condition C and $I^{-}(v) \supseteqq I^{-}(w)$ imply $I^{+}(w) \supseteqq I^{+}(v)$.

1.4. Metric Perturbations. In Fig. 1.2.B, perturbing $g$ slightly may cause $J^{-}(x)$ to increase suddenly to include all $Q$. To analyze such behavior, suppose $\bar{g}$ is another Lorentzian metric on $M . \bar{g}$ is called larger than $g$ if the lightcones of $\bar{g}$ are everywhere broader than those of $g$. Formally, $\bar{g}>g$ iff, for every non-zero vector $V, g(V, V) \leqq 0$ implies $\bar{g}(V, V)<0$. If $z$ is an event in $M$, let $J^{ \pm}(z ; \bar{g})$ and $I^{ \pm}(z ; \bar{g})$ denote the sets formed using $\bar{g}$ rather than $g$. Define the Seifert past $J_{S}^{-}(z)$ as follows: an event is in $J_{S}^{-}(z)$ iff it is in $J^{-}(z ; \bar{g})$ for all metrics $\bar{g}$ larger than $g$. 
Proposition 1.5. (Seifert [14]). For all $z, J_{S}^{-}(z)$ is closed.

Proof. Suppose $w \neq z$ and $w \in$ Closure $\left[J^{-}(z ; \bar{g})\right]$ for all metrics $\bar{g}>g$. For each such $\bar{g}$ choose a $g^{\prime}$ with $\bar{g}>g^{\prime}>g$. Then we Closure $\left[J^{-}\left(z ; g^{\prime}\right)\right]$ so there is a $v \neq w$ such that $v \in$ Closure $\left[J^{-}\left(z ; g^{\prime}\right)\right]$ and that $w \in J^{-}\left(v ; g^{\prime}\right)$ $([9,13])$. We have $v \in$ Closure $\left[J^{-}(z ; \bar{g})\right]$ and $w \in I^{-}(v ; \bar{g}) \subseteq I^{-}(z ; \bar{g})$ $\cong J^{-}(z ; \bar{g})$. Thus

$$
J_{S}^{-}(z)=\bigcap_{\bar{g}>g} J^{-}(z ; \bar{g}) \supseteqq \bigcap_{\bar{g}>g} \text { Closure }\left[J^{-}(z ; \bar{g})\right] \supseteqq \text { Closure }\left[J_{S}^{-}(z)\right] .
$$

In particular, $J_{S}^{-}(z) \supseteqq$ Closure $\left[J^{-}(z)\right]$ for all events $z$. A peculiarity in Fig. 1.2.B is that Closure $\left[J^{-}(z)\right] \neq J_{S}^{-}(x)$, corresponding to a lack of smoothness under small perturbations of the metric.

1.6. Inner and Outer Continuity. In Fig. 1.2.B moving $x$ slightly to the left or up gives a sudden increase in $I^{-}(x)$. To analyze such behavior, suppose $F$ is a function which assigns to each event $z$ in $M$ an open set $F(z)$ in $M . F$ is called inner continuous if, for any $z$ and any compact set $C \subseteq F(z)$, there is an open neighborhood $U$ of $z$ such that $C \subseteq F(u)$ for all events $u$ in $U$. Similarly, $F$ is outer continuous if, for any $z$ and any compact set $K \subseteq M-$ Closure $F(z)$, there is such a neighborhood $U$ with $K \subseteq M-$ Closure $[F(u)]$ for all $u$ in $U$.

Proposition 1.7. (compare [5]). $I^{-}(z)$ is inner continuous.

Proof. Suppose $C \cong I^{-}(z)$ is compact. $\left\{I^{-}(w): w \in I^{-}(z)\right\}$ is an open covering of $C$. Choose $w_{1}, \ldots, w_{n}$ to determine a finite subcovering. Then $\bigcap_{m=1}^{n} I^{+}\left(w_{m}\right)=U$ is an open neighborhood of $z$ with the required property.

In Fig. 1.2.B, $I^{-}(z)$ is not outer continuous. $I^{-}(x) \nsupseteq K$ but in each open neighborhood of $x$ there is a $u$ such that $I^{-}(u) \supseteqq K$.

1.8. Global Time Functions. Let $t$ be a real-valued function on $M$. $t$ is a $C^{0}$ global time function if it is continuous and is monotonic increasing along every future-directed curve. Now it is always possible to find an additive measure $H$ on $M$ which assigns positive volume $H[U]$ to each open set $U$ and assigns finite volume $H[M]$ to $M$ (this result is due to Geroch [5]). Indeed one can find many such measures. Even if $M$ is orientable it may be that none of these are directly related to the Lorentzian volume element $\sqrt{-g} d^{4} x$. Given $H$ we can ask whether setting $t^{ \pm}(z)=\mp H\left[I^{ \pm}(z)\right]$ determines $C^{0}$ global time functions $t^{ \pm}$on $M$. It is intuitively plausible that in Fig. 1.2. B, $t^{-}$will be discontinuous at $x$ no matter how $H$ is chosen. 


\section{Causal Continuity}

A time-oriented spacetime $M$ is (past and future) distinguishing if, for all events $z$ and $w, I^{-}(z)=I^{-}(w)$ or $I^{+}(z)=I^{+}(w)$ implies $z=w$ (compare reference [10], Section 1). A distinguishing spacetime is causal. A causal spacetime need not be distinguishing (for counterexamples see [10] and [9]) but in most intuitive discussions one can regard causality and distinction as essentially equivalent conditions.

Throughout this section $M$ is a time-oriented, distinguishing spacetime. We analyze how the various peculiarities discussed in Section 1 are interrelated. Suppose $t^{+}$and $t^{-}$are as in Subsection 1.8.

Theorem 2.1. The following four conditions are equivalent.
A) $M$ is reflecting;
B) $\mathrm{I}^{+}$und $\mathrm{I}^{-}$are outer continuous;
C) $t^{+}$and $t^{-}$are $C^{0}$ global time functions;
D) $J_{S}^{ \pm}(z)=$ Closure $\left[J^{ \pm}(z)\right]$ for all events $z$.

Proof. A $\rightarrow$ B. Suppose condition A holds and $K \in M-$ Closure $\left[I^{-}(z)\right]$ is compact. If $v \in$ Closure $I^{-}(w)$ for all $w \in I^{+}(z)$ then $v \in$ Closure $\left[I^{-}(z)\right]$ by the argument used in proving Proposition 1.3. Thus

$$
\left\{M \text {-Closure }\left[I^{-}(w)\right]: w \in I^{+}(z)\right\}
$$

is an open covering of $K$. As in Proposition 1.7 we choose a finite subcovering determined by $w_{1}, \ldots, w_{n}$ and $U=\bigcap_{m=1}^{n} I^{-}\left(w_{m}\right)$ is a neighborhood whose existence guarantees outer continuity. Dually, $I^{+}(z)$ is outer continuous.

$\mathrm{B} \rightarrow \mathrm{C}$. Suppose we are given $z \in M$, a measure $H$, and $\varepsilon>0$. Choose a compact $K \cong M-$ Closure $\left[I^{-}(z)\right]$ such that $H\left[M-\right.$ Closure $\left.\left\{I^{-}(z)\right\}-K\right]$ $<\varepsilon$ and choose an open neighborhood $U$ of $z$ such that $K \subseteq M-I^{-}[U]$. Then for all $u \in U, t^{-}(u)-t^{-}(z)<\varepsilon$. Thus $t^{-}$is upper semi-continuous; similarly $t^{-}$is lower semi-continuous by Proposition 1.7. Thus $t^{-}$and, dually, $t^{+}$are continuous. Now suppose there is a future directed curve from $y$ to $x$. Then $I^{-}(x) \supseteqq I^{-}(y)$. Since $M$ is distinguishing, $I^{-}(x)-I^{-}(y)$ is not empty. This implies $I^{-}(x)$-Closure $\left[I^{-}(y)\right]$ is non-empty by the properties quoted in proving Proposition 1.3. Thus $t^{-}(x)>t^{-}(y)$. Dually, $t^{+}$is also monotonically increasing along every future-directed curve.

$\mathrm{C} \rightarrow \mathrm{A}$. Let $\Gamma$ be a smooth, timelike, past-directed curve with past endpoint $z \in M$. For all events $w \in \Gamma-z, I^{-}(w) \supseteqq \downarrow I^{+}(z) \supseteqq I^{-}(z)$. Let $Q=\downarrow I^{+}(z)$ - Closure $\left[I^{-}(z)\right]$. Since $t^{-}$is continuous, $Q$ must be empty. 


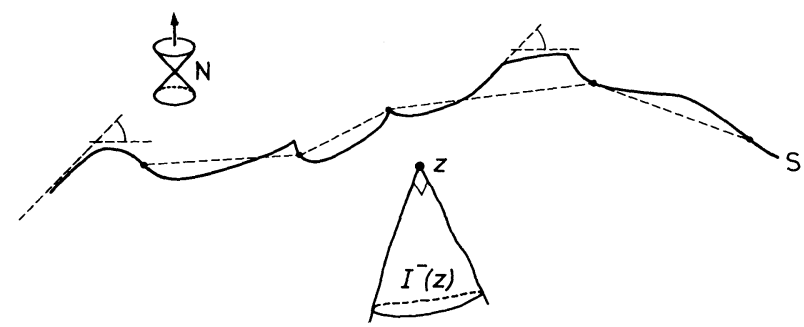

Fig. 2.2. The spacetime $N$, with $I^{-}(z)$ separated from $\dot{I}^{-}(w)$, which corresponds to future infinity in $N$, by $S$

Thus $\downarrow I^{+}(z)=I^{-}(z)$. The dual argument, and Proposition 1.3, show $M$ is reflecting.

$(\mathrm{A}-\mathrm{C}) \rightarrow \mathrm{D}$. Suppose A-C hold, $z \in M$, and $w \in I^{+}(z)$. We will first show that there is a larger metric $\bar{g}$ such that $I^{-}(w) \supseteqq J^{-}(z ; \bar{g})$. $N=I^{-}(w)$ is connected and open, so $N$ is a spacetime. $N$ inherits a time-orientation from $M ; N$ is reflecting since $M$ is reflecting and no future-directed or past-directed curve in $M$ can leave and reenter $N$; similarly, $N$ is distinguishing. Thus we can find a $C^{0}$ global time function $t^{+}$on $N$, as in part $C$. For all $v \in$ Closure $\left[I^{-}(z)\right], t^{+}(v) \leqq t^{+}(z) \equiv a<0$.

Define $S=\left\{v \in N: t^{+}(v)=a / 2\right\}$. $S$ is a boundary of the past set given by $t^{+}<a / 2$ and is therefore a Lipshitz continuous hypersurface $([9,13]$; see Fig. 2.2). No future-directed or past-directed curve intersects $S$ more than once. The idea is to replace $S$ by a piecewise $C^{\infty}$ spacelike hypersurface, as indicated by dashed lines in the figure.

Choose a locally finite, countable covering of $N$ such that each neighbourhood is a geodesically convex normal neighborhood and is relatively compact, while $t^{+}$varies by at most $|a| / 4$ within each neighborhood. Consider a triangulation of $S$ such that each tetrahedron lies within some such neighborhood. If $r, s \in S$ are vertices of the same tetrahedron, the local geodesic from $r$ to $s$ is spacelike. Number all the vertices of the triangulation. For each triangle, with vertices say $r, s, t \in S$, construct the set obtained by drawing local geodesics from that vertex, say $t$, assigned the smallest number to the local geodesic from $r$ to $s$. Construct a geodesic tetrahedron to replace each tetrahedron of the triangulation by similarly drawing geodesics from the vertex assigned the smallest number to the opposite geodesic triangle. For a sufficiently fine triangulation, all the sets thus constructed will be $C^{\infty}$ and spacelike. Each neighborhood of the covering contains only a finite number of the constructed lines, triangles, and tetrahedra. It follows that there is a larger metric $\bar{g}$ on $M$ which leaves all the constructed figures spacelike. For this $\bar{g}, I^{-}(w) \supseteqq J^{-}(z ; \bar{g})$. 
For each $w \in I^{+}(z)$ we can find such a $\bar{g}$. Consequently, Closure $\left[\downarrow I^{+}(z)\right]$ $\supseteqq J_{S}^{-}(z)$. Condition 2.1.D now follows from Proposition 1.3 and the dual argument.

$\mathrm{D} \rightarrow$ A. Suppose 2.1.D holds. Then $x \in$ Closure $\left[J^{+}(y)\right]$ iff $x \in J_{S}^{+}(y)$ iff $y \in J_{S}^{-}(x)$ iff $y \in$ Closure $\left[J^{-}(x)\right]$. By Proposition 1.3, $M$ is reflecting.

Call a time-oriented, distinguishing spacetime causally continuous if it obeys any one of the equivalent conditions in 1.3 or 2.1 . The term is suggested by conditions 2.1.B and 2.1.C. Some of the other conditions can be used to relate causal continuity to standard restrictions. $M$ is called causally simple if, for all events $z, J^{+}(z)$ and $J^{-}(z)$ are closed; it is called stably causal if there is a $\bar{g}>g$ such that $(M, \bar{g})$ is causal $([9])$.

Proposition 2.3. A) A causally simple spacetime is causally continuous;

B) A causally continuous spacetime is stably causal.

Proof. Causal simplicity implies condition 1.3.B and thus causal continuity.

Now suppose condition 2.1.D holds; we will show $M$ is stably causal. $J_{S}^{-}$determines a reflexive partial ordering on $M$ by the rule $y \leqq z$ iff $y \in J_{S}^{-}(z)$, since condition 2.1.D holds and $M$ is distinguishing. Seifert [14] has pointed out that this property of $J_{S}^{-}$is necessary and sufficient for $M$ to be stably causal. That $J_{S}^{-}$determines a reflexive partial ordering when $M$ is stably causal is trivial. We give a modified version of Seifert's proof of the converse.

Throughout the proof: $\left\{U_{m}: m=1,2, \ldots\right\}$ is a countable, locally finite covering of $M$ by relatively compact open neighborhoods $U_{m} ;\left\{f_{m}\right\}$ is a partition of unity subordinate to this covering; $\omega$ is a smooth, everywhere timelike 1 -form on $M ; x, y, z \in M$; and $j, j^{\prime}, j_{x}, j_{y}, j_{z}, j_{m}$ are all smooth functions $M \rightarrow(0, \infty)$. Thus $g-j \omega \otimes \omega$ is a Lorentzian metric on $M$ larger than $g$, etc. We shall need the following two definitions. If $\left(M, g^{\prime}\right)$ is a time-oriented spacetime, $z$ is called causal with respect to $g^{\prime}$ if no curve through $z$ future-directed with respect to $g^{\prime}$ has self-intersections. $z$ is called strongly causal with respect to $g^{\prime}$ if each neighborhood of $z$ contains a neighborhood which no such curve can leave and reenter. If $z$ is strongly causal it is causal, but the converse need not hold; the set of points strongly causal with respect to $g^{\prime}$ is open $([4,13,9])$.

We first show that for some $j_{x}, x$ is a strongly causal point of $\left(M, g_{x}\right)$, where $g_{x}=g-j_{x} \omega \otimes \omega$. Since $J_{S}^{-}$determines a partial ordering, there is some $\bar{g}>g$ such that $x$ is a causal point of $(M, \bar{g})$. Consequently there is some $j$ such that $x$ is a causal point of $(M, g-j \omega \otimes \omega)$. Let $j_{x}=j / 2$. If $x$ were not a strongly causal point of $\left(M, g_{x}\right)$ there would be a $y \neq x$ such that $y \in J^{-}\left(x ; g_{x}\right)$ and $\downarrow I^{+}\left(y ; g_{x}\right) \supseteqq I^{-}\left(x ; g_{x}\right)([10,9,13])$. This would contradict the fact that $x$ is a causal point of $(M, g-j \omega \otimes \omega)$.

We now show that for some $j_{1}$, each point of $U_{1}$ is a (strongly) causal point of $\left(M, g-j_{1} \omega \otimes \omega\right)$. In fact, we can choose a finite number of points 
$x, y, \ldots \in$ Closure $\left[U_{1}\right]$, an open neighborhood $U_{x}, U_{y}, \ldots$ for each, corresponding metrics $g_{x}, g_{y}, \ldots$ and functions $j_{x}, j_{y}, \ldots$ as above such that the open neighborhoods cover Closure $\left[U_{1}\right]$ and each point of $U_{x}$ is strongly causal with respect to $g_{x}$, etc. Choose $j_{1}$ such that $j_{1}<j_{x}$, $j_{1}<j_{y}, \ldots$

Now let $g_{1}=g-\frac{1}{2}\left(f_{1} j_{1}\right) \omega \otimes \omega$. We will show $J_{S}^{-}\left(\cdot ; g_{1}\right)$ determines a partial ordering. There is a $j_{z}$ such that $z$ is a (strongly) causal point of $\left(M, g_{z}\right)$. Take $j^{\prime}$ such that $j^{\prime}<j_{z}$ and $j^{\prime}<\frac{1}{2} j_{1}$ everywhere; define $g^{\prime}=g_{1}-j^{\prime} \omega \otimes \omega$. Then no curve through $z$ which is future-directed with respect to $g^{\prime}$ can have self intersections: if the curve contains a point in $U_{1}$, self intersections are excluded by $j^{\prime}+\frac{1}{2}\left(f_{1} j_{1}\right)<j_{1}$, otherwise they are excluded by $j^{\prime}<j_{z}$. This holds for all $z$, so $J_{S}^{-}\left(\cdot ; g_{1}\right)$ determines a partial ordering.

By induction there are smooth functions $j_{m}$ such that, with $g_{n}=g-\frac{1}{2}\left\{\sum_{1}^{n} f_{m} j_{m}\right\} \omega \otimes \omega,\left(M, g_{n}\right)$ is causal. Let $g^{*}=g-\frac{1}{2}\left\{\sum_{1}^{\infty} j_{m} f_{m}\right\} \omega \otimes \omega$ thus $g^{*}>g$. We will show $\left(M, g^{*}\right)$ is causal. In fact, if $\Gamma$ is a closed curve then $\Gamma$ is compact. Consequently there is an $n<\infty$ such that $U_{m} \cap \Gamma$ is empty for all $m>n$. Let $N$ be that connected component of $\sum_{1}^{n} U_{m}$ which contains $\Gamma$. Then $\left(N, g_{n}\right)$, where $g_{n}$ is restricted to the open submanifold $N$, is a causal spacetime and is an open sub-spacetime of $\left(M, g^{*}\right)$. Thus $\Gamma$ cannot be future-directed in $\left(N, g_{n}\right)$ or in $\left(M, g^{*}\right)$. Since $\left(M, g^{*}\right)$ is causal and $g^{*}>g,(M, g)$ is stably causal.

Both converses are false. The spacetime of Fig. 1.2.A and the maximally extended Reissner-Nordstrom spacetimes (discussed in [9]) are causally continuous but not causally simple. The spacetime of Fig. 1.2.B is stably causal but not causally continuous. The various other restrictions that are sometimes placed on time-oriented spacetimes can be arranged in the following chain $([4,10,9,13])$.

Asymptotically simple and empty $\rightarrow$ globally hyperbolic $\rightarrow$ causally simple $\rightarrow$ causally continuous $\rightarrow$ stably causal $\rightarrow n^{\text {th }}$ order strongly causal $(n=3,4, \ldots) \rightarrow$ strongly causal $\rightarrow$ (future and past) distinguishing $\rightarrow$ future distinguishing or past distinguishing $\rightarrow$ weakly distinguishing $\rightarrow$ causal $\rightarrow$ chronological.

The first four conditions refer primarily to limitations on the kind of gaps spacetime can have and its behavior near infinity. The other conditions refer primarily to near causality violations or actual causality violations.

\section{Conclusion}

In Fig. 1.2.B an observer at $x$ who waits a moment may find all his predictions upset by the new information which comes from the indefinitely large region $Q$. One could argue that such behavior destroys the very 
possibility of doing physics and should be excluded by assuming that $I^{-}(z)$ is continuous. If one wants to make the basic assumptions timesymmetric, one would then also assume $I^{+}(z)$ is continuous. Adding the reasonable requirement that spacetime be distinguishing then implies causal continuity. On the other hand, in 1.2.B the extra information will arrive in a rather diluted form, since it must sneak through a narrow channel to get from $Q$ to a point just above $x$. Thus there is some reason, but no fully convincing argument, for regarding causal continuity as a basic macrophysical property.

The authors are grateful to C. Clarke, E. Kronheimer, R. Melrose, and R. Penrose for discussions.

\section{References}

1. Bishop, R.L., Goldberg, S. I.: Tensor analysis on manifolds, New York: MacMillan 1968

2. Budic, R., Sachs, R. K.: Set causality in general relativity (preprint, 1973)

3. Carter, B.: Phys. Rev. 174, 1559 (1968)

4. Carter, B.: Gen. Rel. Grav. 1, 349 (1971)

5. Geroch, R.: J. Math. Phys. 11, 437 (1970)

6. Geroch, R., Kronheimer, E. H., Penrose, R.: Proc. Roy. Soc. A 327, 545 (1972)

7. Hawking, S.: Proc. Roy. Soc. A 308, 433 (1968)

8. Hawking, S., Penrose, R.: Proc. Roy. Soc. A 314, 529 (1970)

9. Hawking, S., Ellis, G.F.R.: The large scale structure of space-time. Cambridge University Press, 1973

10. Kronheimer, E. H., Penrose, R.: Proc. Cambridge Phil. Soc. 63, 481 (1967)

11. Penrose, R.: Proc. Roy. Soc. A 284, 159 (1965)

12. Penrose, R.: The Structure of Spacetime. In: de Witt, C.M., Wheeler,J.A. (Eds.): Batelle recontres. New York: Benjamin, 1968

13. Penrose, R.: Techniques of differential topology in general relativity. Am. Math. Soc. Colloq. Publ., 1973

14. Seifert, H.: Gen. Rel. Grav. 1, 3, 247 (1971)

15. Woodhouse, N. M. J.: J. Math. Phys. 14, 4, 495 (1973)

S. W. Hawking

Institute of Astronomy

University of Cambridge

Cambridge, U.K.

R. K. Sachs

DAMTP

University of Cambridge

and

Departments of Mathematics and Physics

University of California at Berkeley

Berkeley, Cal. 94720, USA 